\title{
GRB 011211: An alternative interpretation of the optical and X-ray spectra in terms of blueshifts
}

\section{Basu ${ }^{1}$}

Abstract The redshift of the gamma ray burst (GRB) GRB 011211 has been determined as 2.14 from several absorption lines seen in the spectrum of its optical afterglow. The spectrum of its X-ray afterglow exhibited several emission lines, and their identification led to a mean redshift 1.862. A supernova model has been proposed based on the redshift of the GRB as 2.141. It is shown here that the redshift interpretation cannot explain the observed spectra, as some serious inconsistencies exist in the process of redshift determinations in spectra of both optical and X-ray afterglows. In view of that, an alternative interpretation of the spectra is presented in terms of blueshifts. Ejection mechanism is proposed as a possible scenario to explain the blueshifted spectrum.

PACS Nos.: 98.38.Mz: 98.62Py; 98.80.-K

\footnotetext{
${ }^{1}$ Department of Physics, Carleton University, Ottawa, ON K1S 5B6, Canada. e-mail: basu@physics.carleton.ca
} 


\section{Introduction}

Although the first gamma ray burst (GRB) was discovered over thirty years ago [1], it is only in recent years that redshifts are being determined after their optical counterparts are associated with host galaxies. As a result, GRBs are considered as extragalactic objects due to their high redshift values. However, high redshifts imply enormous distances and this has raised the problem of energetics. Meanwhile, several models have been proposed to account for the extremely high energies required, and involve merging of neutron stars (or black holes) in a binary [2], or collapse of massive stars producing black holes with superstrong $\left(\approx 10^{15} \mathrm{G}\right)$ magnetic fields (supernova (SN)/hypernova $(\mathrm{HN})$ models $[3,4]$.

GRB 011211 was detected [5] by the BEPPO-SAX satellite on 11 December 2001 and its redshift was determined [6,7] from several absorption lines seen in the spectrum of its optical afterglow as $\mathrm{z}_{r(o p, a b s)}=2.14$. A new absorption spectrum of the object with $\mathrm{S} / \mathrm{N}$ superior to that of Holland et al. [7] has recently been published [8], and this is quite different from the earlier one with only four common features. Also, as Vreeswijk et al. [8] points out, some of the lines in Holland et al. [7] were actually misidentified. The mean absorption redshift obtained by Vreeswijk et al. [8] is 2.1418 based on the identification of sixteen out of the seventeen lines seen in the spectrum and originating in a single absorption system. 
GRB011211 was subsequently observed by the XMM-Newton X-ray telescope and the spectrum of the X-ray afterglow exhibited "an apparent absorption feature" and five emission lines [9]. The former was identified on the assumption that it "arises in the same material as the line emission", while the identification of the five emission lines with highly ionized metal lines led to a mean redshift $\mathrm{z}_{r(x, e m)}=1.862$. Reeves et al. [10] have, of course, re-analysed their data, and have withdrawn the absorption line which is not real.

Nevertheless, there appears to be some controversy over the analysis of the X-ray spectrum by Reeves et al. [9,10]. Borozdin \& Trudolyubov [11] analyzed the first $5 \mathrm{ksec}$ of the Reeves et al. $[9,10]$ data for both PN alone and combined PN, MOS1 and MOS2. A good fit for an absorbed power law with Galactic absorption was found for the combined data showing no improvement from adding lines. But for the PN data alone, which the Reeves et al. $[9,10]$ analysis is based on, improvement to the fit at 99.9 percent confidence level was found when lines identified as partially ionized lines of $\mathrm{Mg}, \mathrm{Si}, \mathrm{S}, \mathrm{Ar}$, Ca were added with a mean redshift of 1.9. On the other hand, independant analyses by Rutledge \& Sako [12] and Sako et al. [13] suggested the statistical significance of the features to be marginal.

But Butler et al. [14] demonstrated that the conflicting estimation of the statistical significance by Reeves et al. $[9,10]$, and by Rutledge and Sako [12] and Sako et al. [13], has arisen from different assumptions in 
the continuum modelling. Both methods would agree that the lines are statistically significant at $\approx 3 \sigma$ level, as originally claimed by Reeves et al. [9], if the column density is taken as the Galactic value as in the analysis of Reeves et al. [9]. Butler et al. [14] have further shown that the density parameter should actually be taken as the Galactic value for the first 5ksec portion of the XMM-Newton EPIC-PN spectrum where the potential emission lines are located, since the assumption of the Galactic absorption leads to well modelling of the full $25 \mathrm{ksec}$ data. This confirms that the emission lines reported by Reeves et al. $[9,10]$ are real and statistically significant.

The importance of the correct estimation of redshifts in building models of extragalactic objects, including GRBs, can hardly be underestimated, as redshifts are essential for determining the energetics of the objects. The model proposed $[9,10]$ for the GRB 011211 is an SN model, the computed total equivalent isotropic energy of $5 \times 10^{52}$ erg being based on the redshift 2.141 .

One has to keep in mind that all line identification processes are programmed, almost as a rule, to determine redshifts only, by identifying search lines to observed lines at the red side. No attempt is made to identify search lines with observed lines at the blue side, and, as such, blueshifts are not considered. Possibility of misidentification of observed lines, and, hence, of blueshifts in spectral lines of extragalactic objects have, of course, been pointed out by several researchers in the past [15-17] Observed lines are in- 
deed sometimes misidentified for redshift determinations. Spectra of objects like GRB 011211 that cannot be explained in terms of redshifts, should be particularly considered and re-examined in this respect. The purpose of this paper is to show that an alternartive interpretation in terms of blueshifts can explain the spectra.

Sec. 2 reviews some specific examples in the literature to demonstrate misidentifications of observed lines and their possible blueshift $\left(\mathrm{z}_{b}\right)$ interpretations. Serious inconsistencies in the redshift $\left(\mathrm{z}_{r}\right)$ deteremination in the spectra of both optical and x-ray afterglows of GRB 011211 are demonstrated in Sec. 3, and the spectra is interpreted in terms of the alternative blueshift hypothesis in Sec. 4. Ejection mechanism is proposed as a possible scenario to explain the blueshifted spectra in Sec. 5. and some concluding remarks are presented in Sec. 6 .

\section{Examples of misidentification of lines and possible blueshifts}

It is known that CIV 1549 and CIII] 1909 are two of the strongest search lines in the redshift identification system, and if one of them is seen the other should be seen as well in the observed spectrum [18]. However, published spectra in the high redshift galaxy $(\mathrm{HRG})$ 0316-257B $\left(\mathrm{z}_{r}=3.1351\right)$ exhibits CIV, but there is no line at $7894 \AA$, the expected position of CIII] [19], and the authors do not even mention about the CIII]. Same is the case with another HRG Obj $19\left(\mathrm{z}_{r}=2.39\right)$ which exhibits CIV [20], and a very insignificant 
and most like likely noise signal is claimed as the CIII] at the expected wavelength of $6472 \AA$, marked doubtful. The spectra of these two and another 13 HRGs have been explained by re-identifications of observed lines in terms of blueshifts [21].

Again, slitless spectroscopy of the galaxy STIS123627+621755 demonstrates [22] an emission line around $9334 \AA$, followed by a discontinuity starting around $9300 \AA . A z_{r}=6.68$ was determined by the identification of the line with Ly $\alpha 1216$ and the discontinuity as the Ly decrement. However, subsequent observation at $\mathrm{B}, \mathrm{V}$ bands $[23]$ found the identifications wrong and concluded that "the redshift is undetermined", as Ly $\alpha$ limit (912A) is shifted to $7004 \AA$ at $\mathrm{z}_{r}=6.68$ and no flux should be observed below $7004 \AA$, i.e. at $\mathrm{B}, \mathrm{V}$ bands. The same galaxy was also observed at $6700 \AA$ And a non-detection was reported at 1.2 micron [24] which also ruled out the $\mathrm{z}_{r}$ of 6.68 . The spectra has been successfully interpreted as blueshifted [25].

Furthermore, the spectrum of the QSO PG $1407+265$ is very unusual in the sense that the major UV lines viz. Ly $\alpha$, CIV, CIII, MGII 2798, are very weak and $\mathrm{H} \alpha$ is also much weaker than normal, while the usually weak FeII forest [26] are "unusually strong" [27]. Several attempts to explain the spectrum turned unconvincing and the nature of the spectrum "remains puzzling" [27].

In addition, the spectrum of PG $1407+265$ also exhibits a large number of absorption lines leading to the determination of several absorption redshift 
systems [28]. However, the identifications of the absorption lines show many inconsistencies. Some lines remain unidentified, several identifications are reported doubtful, the same search line $(\operatorname{Ly} \alpha)$ has been identified with more than one observed lines for the same redshift system in many cases, several redshifts have ben computed by identification of a single line, 'spread' values are larger than the usually accepted value of 0.01 for absorption redshift syatems in most cases, a redshift system has been computed on the basis of identification of a higher order Lyman line with a stronger observed line and a lower order Lyman line with a weaker observed line. All these discrepancies and inconsistencies lead to the absorption redshift systems unacceptable.

PG 1407+265 and unusual spectra of two other QSOs, viz. SDSS 1533-00 and PKS 0637-752 have been explained on the basis of blueshift hypothesis [29]. Additionally, observed spectra of 25 other QSOs available in the published literature have also been identified with search lines of longer wavelengths and blueshifts determined [30]. The spectra of another QSO (radio loud), viz. PKS 2149-306, which could not be explained in the usual redshift interpretation has been successfully re-interpreted as blueshifted [31].

Moreover, QSO pairs seen across active galaxies are believed to be ejected from the galaxy involved. It is, however, more logical that the pair should be ejected in opposite directions with equal probability, and, as such, one should be moving away from us exhibiting redshift, while the other should be approaching us exhibiting blueshift, rather than both exhibiting redshifts 
implying both are ejected away from the observer. Analysis of four pairs demonstrated that the observed spectrum of one object in each pair can be interpreted as blueshifted [32].

Further, the redshift of the host galaxy of the GRB 971214 has been eveluated by the identification of one emission line and "a drop immediately on the blue (short wavelength) side of the line", in addition to the absence of any flux "blueward of 4030 Åthe redshifted Ly $\alpha$ continuum break" [33]. However, the published record shows that the 'drop' is not acceptable as its magnitude is of the same order as the noise level. On the other hand, the record stops at $4000 \AA$ which makes the argument of "no flux blueward of $4030 \AA "$ unconvincing. The spectra the host galaxy of 971214 and three other GRB host galaxies have been interpreted as blueshifted [34].

Again, the observed spectrum of the host galaxy of the SN Ia 96T exhibits three emission features identified in the redshift scenario as $\mathrm{H} \alpha$, [OIII] 5007 and [OII] 3727 [35]. However, examination of the profiles of the lines revealed that the lines at $8141 \AA$ and $6212 \AA$ identified with $\mathrm{H} \alpha$ and [OIII] 5007 respectively are unacceptably week for the two recognized strong search lines [30], actually weaker than the line at $4626 \AA$, identified with the [OII] 3727 (unfortunately, no equivalent widths are avilable). The spectra of the host galaxy of $96 \mathrm{~T}$ and host galaxies of four other $\mathrm{SNe}$ Ia were re-interpreted as blueshifted, and blueshifts determined by re-identification of the observed lines with search lines of longer wavelengths [36]. 
Finally, the puzzling spectrum of the galactic X-ray source 1E 1207.45209 has been explained as blueshifted and shown to be due to two ejected absorbing clouds originating at the centre of the SNR G296.5+10.0 [37].

\section{Inconsistencies in the redshift determination of GRB 011211}

\subsection{Optical absorption lines}

The assumption that the redshift of the GRB is equivalent to $\mathrm{z}_{r(o p, a b s)}=$ 2.141 implies that all the absorption lines in the optical spectrum arise in the host galaxy [10]. In reality, this is the lower limit of the GRB redshift. The redshift of an extragalactic object is the emission redshift determined from the emission lines. Radiation from the object is more likely to encounter several absorbing clouds in the line of sight between the object itself and the observer. Redshifts determined from absorption lines arising in these clouds, i.e. absorption redshifts, are therefore, in general, smaller than the redshift of the object if redshifts are cosmological. The number of absorption redshift systems for an extragalactic object with the redshift around 2.2 may be as high as 20 [38]. However, some lines may arise in the host galaxy, in which case the largest of the absorption redshifts will be equal to the redshift of the host galaxy, the latter being determined, once again, from emission lines. Hence, more than one absorption redshift systems, all smaller than (although one may be equal to) the emission redshift, is expected. Spectra 
with different features, viz. emission and absorption, have been reported for several other GRBs, as discussed below, which would support the scenario presented above.

GRB 021004 exhibits many absorption lines that have been identified with search lines leading to as many as five absorption systems, viz. 1.3806, 1.6039, 2.2983, 2.3230 and 2.3293, while the single emission line identified with Ly 1216 yields the emission redshift 2.3351 [39]. Thus, the largest absorption system is nearly equal to the emission system, i.e. the redshift of the host galaxy, with a difference of only 0.0058 . This makes the largest system arising in the host galaxy itself, and the other absorption systems, smaller than the emission system, arising in the intervening absorbing clouds under cosmological hypothesis [39]. These redshifts are also confirmed by Mirabal et al. [40], who equate the absorption redshift 2.328 of the AlII 1670.71 line to the Ly $\alpha 1216$ emission redshift 2.328, computed from their data. and concludes that this highest system originates in the host galaxy.

Another object, viz. GRB 020405, was detected at radio and X-ray wavelengths $[41,42]$, although it did not exhibit any discrete feature in its X-ray spectrum, neither emission nor absorption. This has been interpreted as the effect of a "long lasting bright afterglow" that might have been responsible for the non-detection of any faint discrete feature [41]. Nonetheless, the optical spectrum of the host galaxy exhibited Balmer and oxygen emission lines which yielded the redshift 0.691 [43]. Additionally, the optical spectrum is 
also rich in absorption lines, showing twelve features which have been identified with FeII and MgII lines, yielding two absorption systems, viz. 0.691 and 0.472. Once again, the former, the larger of the two, being equal to the emission system, originates in the host galaxy itself, and the latter is an intervening system identified by the imaging technique with a cloud in the galaxy complex [43].

It thus appears that spectra of GRB host galaxies, rich in absorption lines and also exhibiting emission features, is not uncommon. In such cases, the absorption lines are interpreted as multiple absorption redshift systems with the largest, if and when having a similar value as the emission redshift system, originating in the host galaxy, and others, having smaller values, originating in intervening space. Apart from the papers quoted above, reference can also be made to $[44,45]$, where presence of intervening systems have been reported for the GRB host galaxy spectra.

In case of the present object, viz. GRB 011211, the assumption that the redshift of the object is equal to a single absorption redshift exhibited by all the absorption lines, viz. as many as seventeen, is an oversimplification of the situation. Therefore, even if the redshift scenario is correct, the redshift of the GRB 011211 is most likely much larger than 2.141. This puts the GRB at a much larger distance, and hence involves much larger energy than the energy of a typical supernova, viz. $5 \times 10^{52} \mathrm{erg}$, which the model is based on.

The importance of the above arguments is further evident in the seri- 
ous inconsistency in the determination of the absorption redshift $\mathrm{z}_{r(o p, a b s)}=$ 2.1418 [8], which cannot identify all the lines in the spectrum, the line at 6114.2Åremaining unidentified. This clearly shows that a single system has failed to explain the observed absorption spectrum in the redshift interpretation. A redshift system cannot be accepted unless all the lines in the system are identified exhibiting the same redshift value.

The improbability of having a single absorption system to explain all the absorption lines in an object exhibiting a redshift around 2.2, as discussed above, and the fact that the system cannot even identify all the lines in the observed spectrum, lead to the conclusion that the redshift cannot be accepted as the redshift of the host galaxy.

\subsection{X-ray emission lines}

The X-ray spectrum exhibits five emission lines which have been identified to "the closest abundant K $\alpha$ transitions to the observed lines", based on the redshift $\mathrm{z}_{r(o p, a b s)}=2.14[9,10]$. The mean redshift is $\mathrm{z}_{r(x, e m)}=\mathrm{z}_{r}$ $=$ redshift of the host galaxy of the GRB $011211=1.862$, computed from these identifications. This does not match at all with the optical absorption redshift, and the difference $\mathrm{z}_{r(o p, a b s)}-\mathrm{z}_{r}=0.278$, implying an outflow velocity $(\mathrm{v})$ for the line emitting material of $\mathrm{v} / \mathrm{c}=0.085 \pm 0.02\left(\approx 2.5 \times 10^{4}\right.$ $\mathrm{kms}^{-1}$ ), where $\mathrm{c}$ is the velocity of light. Thus, some "arbitrary blueshifts are 
invoked to adjust the closest atomic transition to match the observed energy of detected excesses" [13].

Furthermore, the mean redshift of the five emission lines in the X-ray spectrum is, as mentioned above, $\mathrm{z}_{r}=1.862$, but the difference ( $\operatorname{spread} \Delta \mathrm{z}_{r}$ ) between the maximum redshift of the system 2.03 exhibited by Mg XI and the minimum redshift of the system 1.73 exhibited by Ar XVIII is $\Delta \mathrm{z}_{r}=$ 0.3 (see Table 1). The alternative identification of Mg XII instead of MgXI, suggested by the authors $[9,10]$, is actually more appropriate for a Ly $\alpha$-like transition, but then, the redshift is 2.3409 and $\Delta \mathrm{z}_{r}=0.6109$. No reason has been given by these authors for not adopting this alternative identification.

Ideally, spread values should be close to zero. However, upto a certain extent, spreads can have some physical reasons, mainly because of the difficulty encountered in the exact determination of the observed wavelength. The latter, in its turn, may have reasons of its own, viz. the profile being broad or double- or multi-peaked or of complex structure, blending, intrinsic or intervening absorption, gradients, net flows, partial screening, etc. The unacceptably high value $\Delta \mathrm{z}_{r}=0.3$, let alone $\Delta \mathrm{z}_{r}=0.6109$, cannot be explained by any physical mechanism.

\section{The alternative blueshift determination in GRB 011211}

The blueshift $\left(\mathrm{z}_{b}\right)$ of an object is determined by the relation $\mathrm{z}_{b}=\left(\lambda_{e^{-}}\right.$ 
$\left.\lambda_{o}\right) / \lambda_{e}$, where $\lambda_{e}$ and $\lambda_{o}$ are emitted and observed wavelengths respectively in $\AA$. $\lambda_{e}$ is obtained from the search list of known laboratory lines. An extended search list covering the UV, optical and IR regions has been prepared and is available in [30]. $\lambda_{o}$ is obtained from the record of observation. In the X-ray region, where wavelengths are usually expressed in energy units, the blueshift is determined by $\mathrm{z}_{b}=\left(\mathrm{E}_{o}-\mathrm{E}_{e}\right) / \mathrm{E}_{o}$, where $\mathrm{E}_{e}$ and $\mathrm{E}_{o}$ are emitted and observed values of wavelengths respectively in $\mathrm{keV} . \mathrm{E}_{e}$ is available in standard tabls and $\mathrm{E}_{o}$ is obtained from the observed spectra.

We have interpreted both the optical absorption spectrum (current superior quality data of Vreeswijk et al. [8]) and the X-ray emission spectrum, in terms of blueshifts. Table 1 shows the identifications of the observed lines in the spectra of GRB 011211 in both redshift and blueshift interpretations. We have followed the standard procedure in the identification process $[46,47]$, viz. a 'shift' (red or blue) is only confirmed when at least two observed lines, emission spectrum or absorption spectrum, exhibit the same value when identified with two separate search lines. Any third or more lines seen in the spectrum, and, in case of absorption lines, belonging to the same system, have also to obey the same value. Further, in our identification, if and when, the lower order line(s) of a series and/or the stronger component of a doublet are/is identified, the higher order line(s) of the series and/or the weaker component of the doublet may be too weak to be seen. If and when, however, the higher order line(s) of a series and/or the weaker component 
of a doublet are/is identified, the lower order line(s) of the series and/or the stronger component of the doublet are/is outside the observed region of the spectrum.

For the optical spectra, all the observed lines have been identified with alternative search lines of longer wavelengths, including the line at $6114.2 \AA$ which the redshift hypothesis failed to identify. In the blueshift identification, the seventeen absorption lines seen in the optical spectrum are absorbed in six separate clouds representing six separate systems, viz. (i) $\mathrm{H} \alpha 6563$, HeI 7065, OI 8449 (ii) OI 8449, HeII 10124, OI 11210 (iii) OI 8449, HeII 10124, $\mathrm{P} \beta$ 12818 (iv) OI 11210, $\mathrm{P} \beta$ 12818, $\mathrm{P} \alpha 18751$ (v) H2 19750, H2 21218, H2 21542 (vi) HeI 17008, $\mathrm{P} \alpha 18751$.

It may be noted that all these are well recognized search lines used regularly in redshift identification programs as well. Further, all the blueshifted lines are permitted lines to match the observed broad lines and chosen also to match the strengths of the observed lines as given by the equivalent widths. The line at 3820 Aidentified in the redshift system as Ly $\alpha$ yields the rest frame equivalent width of $5.28 \AA$ which is too small as it is known as one of the strongest search lines. The blueshift identification, on the other hand, yields the $\mathrm{H} \alpha$ rest frame equivalent width of 28.52 which matches the strength of the observed line. Same is true for the lines at $4863.4 \AA$ and $4870.9 \AA$ inhich have been identified in the redshift scenario with CIV, another of the strongest search lines in the redshift scenario, yielding rest frame equivalent widths of 


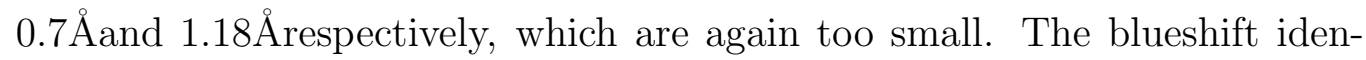
tifications of these lines with medium strong search lines OI 8449 and HeII 10124 [30] yielding rest frame equivalent widths of 3.83 and 7.69 respectively are, once again better fits.

Furthermore, the line at 6114.2 remains unidentified in the redshift system, and the blueshift system has duly identified the line fitting it with other lines of one of the systems.

Again, SiIV 1393/1402 is a medium strong line [30], and its equivalent width of 0.92/0.64 is much smaller than expected. These lines at 4318.7/4405.0 identified respectively with OI 8449 and OI 11210 yielding rest frame equivalent widths of 5.59/5.09 are certainly better matches.

It is worth furher noting that redshifts and blueshifts are determined by identifying the principal line(s) first and other lines then follow matching the redshift or blueshift value(s) thus obtained. As pointed out above, the redshift hypothesis has failed to identify properly the most prominent feature in the absorption spectrum, viz. the line at $3829.0 \AA$.

On the other hand, the six absorption systems have mean blueshift values $z_{b(o p, a b s)} 0.4208,0.4809,0.5249,0.6042,0.6205,0.7487$, with corresponding spreads, $\Delta \mathrm{z}_{b(o p, a b s)}$, calculated as the difference between the maximum and minimum blueshift values of each system, 0.0065, 0.0013, 0.0139, 0.0062, $0.0070,0.0046$ respectively. Unfortunately, uncertainties in the determination of the observed wavelengths $\left(\lambda_{o}\right)$ for the optical absorption lines are not 
available. But published literature would confirm that, usually, for absorption lines in extragalactic objects, $\Delta \mathrm{z}_{r(o p, a b s)}<0.01$. With one exception, viz. the system $\mathrm{z}_{b(o p, a b s)}=0.5249$, with $\Delta \mathrm{z}_{b(o p, a b s)}=0.0139$, all values are within this limit. Such exceptions are, of course, not unheard of in the redshift literature. The optical absorption redshift system 2.8102 in the QSO 0528-250 has a spread of $0.0173[48,49]$.

The five emission lines in the X-ray spectrum are identified with $\mathrm{K} \alpha$ transitions of $\mathrm{Ne}, \mathrm{O}, \mathrm{N}$ and $\mathrm{C}$, and $\mathrm{L} \alpha$ transition of $\mathrm{Fe}$, and the blueshift of each line is determined. The uncertainty in the determination of the blueshift of each X-ray line is also shown in Table 1, based on the uncertainty in the determination of each observed wavelength. The identifications yield $z_{b(x, e m)}$ $=\mathrm{z}_{b}=0.4115$, the mean blueshift of the host galaxy of the GRB 011211, and $\Delta \mathrm{z}_{b}=0.0768 \pm 0.073$. This spread is consistent with $\Delta \mathrm{z}_{b}=0$ at the confidence level of $\sigma=1.05$.

Also, the six elements, viz. Ne, O, N. C, H and Fe are among the most abundant elements in the universe, and are often identified in extragalactic objects, and the $\mathrm{K} \alpha$ transition is the strongest transition in X-ray spectra.

Moreover, the detection of Fe L $\alpha$ has been reported earlier [50] in galaxies M 82 and NGC 253, where Fe K $\alpha$ is very weak (doubtful) or not seen at all. At the blueshift 0.4174 for the $\mathrm{Fe} \mathrm{L} \alpha, \mathrm{Fe} \mathrm{K} \alpha$ is expected $\approx 10.1 \mathrm{keV}$, which is outside the observed wavelength range of Reeves et al $[9,10]$.

The six optical absorption systems with blueshifts larger than that of the 
host galaxy are located in the intervening space along the line of sight. As discussed earlier (Sec. 3.1), this is the expected scenario rather than all the lines being absorbed in the host galaxy, and no absorbing cloud encountered in the intervening path. We propose that the host galaxy, along with the six associated absorbing clouds, have been ejected, as described in the following scenario (Sec. 5).

\section{A generic proposal: ejection mechanism}

It is known that supermassive black holes are seats of activities at centres of galaxies $[51,52]$. When the system becomes gravitationally unstable due to strong interactions at the centre, one or more massive objects may be ejected by the so called "sling-shot" mechanism [53-55]. The scenario has been further developed as follows.

Two galaxies, each hosting a supermassive black hole may merge resulting in the initial formation of a binary system containing the two central black holes [56]. Binary black hole systems have indeed been detected at X-ray wavelengths in NGC 6240 [57], possibly in OJ 287 [58], and very recently in

SDSS J153636.22+044127.0 [59]. As the merger process proceeds further, a single black hole is ejected at a relativistic or non-relativistic speed, if the two individual black holes are of unequal masses [60]. Evidence of ejection of a supermassive black hole by the "sling shot" mechanism resulting from 
merger of galaxies has recently been presented by Haehnelt et al. [61]. Again, it is believed [62-64] that the black hole seated at the centre of a galaxy is often surrounded by a gaseous accretion disk which survives the tidal disruption involved in the ejection process. Several authors have shown that the interaction between the surroundings and the disk associated with the black hole may be responsible for the production of galaxy-like objects [65$67]$.

It is also known that the central supermassive black holes (primaries) may be accompanied by satellite black holes of intermediate masses [68], and a "small black hole swarm around the supermassive black hole in the core of the Milky Way" has recently been reported by Munro (AAS meeting January 2005, Sky \& Telescope April 2005). Satellite black holes are ejected too as a result of the merger process, and at least some of them may assume eccentric orbits around the primary [67].

It is reasonable to assume that satellite black holes are also surrounded by similar gaseous disks, and would undergo similar interactions with their surroundings, as in primaries, although at reduced scales, being of smaller masses, and would end up as faint or nascent or smaller galaxies. The final result of the merger of two galaxies is, therefore, the ejection of a new galaxy, along with several galaxy-like objects. The latter acts as absorbing clouds when falling along the line of sight, and, being ejected at larger speeds, exhibit larger blueshifts. It may be noted in this connection that Basu [38] had 
shown earlier that the appearance of observing clouds in an extragalactic object may be associated with the creation of the object itself. Moreover, ejection mechanism is well known in the literature, and observations [69-71] support such systems, viz. galaxies associted with possible absorbers, the latter being in forms of other galaxies, faint and nascent galaxies.

\section{Concluding remarks}

All extragalactic objects do not exhibit blueshifted spectra. As such, blueshifts do not contradict redshifts, but complement them. Modern observational technology is leading to the discovery of larger number of objects and it appears that some spectra cannot be interpreted as redshifted. While several researchers suggested that blueshifts are possible, observed spectra are routinely interpreted in terms of redshifts only. Considering its possible impact on modern cosmology, possibility of blueshifts should be included in current line identification programs.

Acknowledgement: The author is grateful to an anonymous referee for making helpful comments and suggestions that led to major improvement of the paper. 
Table 1 Redshifts and blueshifts in the spectra of the GRB 011211

\begin{tabular}{|c|c|c|c|c|c|c|c|c|}
\hline TYPE & $\lambda_{o}$ & $\mathrm{~W}_{o}$ & $\mathrm{z}_{r}$ line & $\mathrm{Z}_{r}$ & $\mathrm{~W}_{e r}$ & $\mathrm{z}_{b}$ line & $\mathrm{z}_{b}$ & $\mathrm{~W}_{e b}$ \\
\hline \multirow{18}{*}{ ABS } & 3820.0 & 16.6 & Ly $\alpha 1216$ & 2.1423 & 5.28 & $\mathrm{H} \alpha 6563$ & 0.4179 & 28.52 \\
\hline & 3957.0 & 4.7 & SiII 1260 & 2.1394 & 1.50 & OI 8449 & 0.5328 & 10.06 \\
\hline & & & SiII 1259 & & & & & \\
\hline & 4090.9 & 4.6 & $\begin{array}{l}\text { SIII } 1304 \\
\text { OI } 1302\end{array}$ & 2.1419 & 1.40 & Hell rob5 & 0.4201 & 6.93 \\
\hline & 4195.6 & 5.6 & $\begin{array}{l}\text { CII } 1334 \\
\text { CII } 1335\end{array}$ & 2.1439 & 1.78 & HeI 17008 & 0.7533 & 22.7 \\
\hline & 4318.7 & 2.9 & SiIV 1393 & 2.1438 & 0.92 & OI 8449 & 0.4814 & 5.59 \\
\hline & 4405.9 & 2.0 & SiIV 1402 & 2.1409 & 0.64 & OI 11210 & 0.6070 & 5.09 \\
\hline & 4797.8 & 3.6 & SiII 1526 & 2.1426 & 1.15 & $\mathrm{P} \alpha 18751$ & 0.7441 & 14.07 \\
\hline & 4863.4 & 2.2 & CIV 1548 & 2.1413 & 0.70 & OI 8449 & 0.4244 & 3.82 \\
\hline & 4870.9 & 3.7 & CIV 1550 & 2.1410 & 1.18 & HeII 10124 & 0.5189 & 7.69 \\
\hline & 5053.7 & 1.7 & FeII 1608 & 2.1420 & 0.54 & $\mathrm{P} \beta 12818$ & 0.6057 & 4.30 \\
\hline & 5251.7 & 3.5 & AlIII 1670 & 2.1432 & 1.11 & HeII 10124 & 0.4813 & 6.75 \\
\hline & 5828.3 & 1.7 & AlIII 1854 & 2.1424 & 0.54 & OI 11210 & 0.4801 & 3.27 \\
\hline & 6114.2 & 1.3 & $?$ & $?$ & $?$ & $\mathrm{P} \beta 12818$ & 0.5230 & 2.73 \\
\hline & 7362.8 & 3.1 & FeII 2344 & 2.1408 & 0.99 & H2 19570 & 0.6238 & 8.24 \\
\hline & 7485.5 & 6.5 & FeII 2382 & 2.1415 & 2.07 & $\mathrm{P} \alpha 18751$ & 0.6008 & 16.28 \\
\hline & 8131.4 & 3.8 & FeII 2586 & 2.1436 & 1.21 & H2 21218 & 0.6168 & 9.92 \\
\hline & 8171.4 & 3.5 & FeII 2600 & 2.1426 & 1.11 & H2 21542 & 0.6208 & 9.23 \\
\hline \multirow[t]{6}{*}{ EM } & $0.44 \pm 0.04$ & 0.54 & MgXI 1.35 & 2.03 & 0.18 & CK $\alpha 0.277$ & $0.3705 \pm 0.057$ & 0.86 \\
\hline & & 0.59 & MgXII 1.47 & 2.3409 & & & & \\
\hline & $0.71 \pm 0.02$ & 1.23 & SiXIV 1.99 & 1.82 & 0.43 & NK $\alpha 0.3924$ & $0.4473 \pm 0.016$ & 2.22 \\
\hline & $0.88 \pm 0.01$ & 1.41 & SXVI 2.6 & 1.9438 & 0.48 & OK $\alpha 0.5249$ & $0.4035 \pm 0.007$ & 2.37 \\
\hline & $1.21 \pm 0.02$ & 1.26 & ArXVIII3.3 & 1.73 & 0.46 & FeL $\alpha 0.705$ & $0.4174 \pm 0.01$ & 2.17 \\
\hline & $1.46 \pm 0.04$ & 1.03 & CaXX 4.07 & 1.79 & 0.36 & NeK $\alpha 0.8486$ & $0.4188 \pm 0.016$ & 1.76 \\
\hline
\end{tabular}

TYPE denotes absorption (ABS) or emission (EM) features. $\lambda_{o}$ and $\mathrm{W}_{o}$ are observed wavelength and observed equivalent width, $\mathrm{W}_{e r}$ and $\mathrm{W}_{e b}$ are emitted equivalent widths based on redshifts and blueshifts, $z_{r}$ line and $z_{b}$ line are search lines $\left(\lambda_{e}\right.$ or $\left.\mathrm{E}_{e}\right)$ identified in redshift and blueshift scenarios, $\mathrm{z}_{r}$ and $\mathrm{z}_{b}$ are redshift and blueshift values, respectively.

Top panel, optical spectrum [8], with $\lambda_{o}$ and all W's in $\AA$.

Bottom panel, X-ray spectrum with $\lambda_{o}$ and all W's in keV. $\lambda_{o}, \mathrm{z}_{r}$ line, $\mathrm{z}_{r}$ 
from [10], $\mathrm{W}_{e r}$ from [9]. 


\section{References}

1. Klebasadel, R. et al. Astrophys. J. 182, L85 (1973).

2. Liang, E. et al. Astrophys. J. 479, L35 (1997).

3. Paczynski, B. Astrophys. J. 494, L45 (1998).

4. Iwamoto, K. et al. Nature 395, 672 (1998).

5. Frontera, F. et al. GCN GRB Obs. Rep. No. 1215 (2001).

6. Fruchter, A. et al. GCN GRB Obs. Rep. No. 1200 (2001).

7. Holland, S. et al. Astron. J. 124, 639 (2002).

8. Vreeswijk, P. et al. Astron. Astrophys. 447, 145 (2006).

9. Reeves, J. et al. Nature 416, 512 (2002).

10. Reeves, J. et al. Astron. Astrophys. 403, 463 (2003).

11. Borozdin, K. N and Trudolyobov, S. P. Astrophys. J. 583, L57 (2003).

12. Rutledge, R. E. and Sako, M. Mon. Not. Roy. astyron. Soc. 339, 600.

13. Sako, M. et al. Astrophys. J. 623, 973 (2005).

14. Butler, N. et al. Astrophys. J. 627, L9 (2005).

15. Putsil'nik, S. Astron. Astrophys. 78, 284 (1979).

16. Gordon, K. Amer. J. Phys. 48, 524 (1980).

17. Popowski, P. and Weinzriel, W. Mon. Not. Roy. Astron. Soc. 348, 235 (2004).

18. Weymann, R, et al. Ann. Rev. Astron. Astrophys. 19, 41 (1981).

19. La Fevre, O. et al. Astrophys. J. 471, L11 (1996).

20. Pascarelle, S. et al. Nature 383, 45 (1996). 
21. Basu, D. Astrophys. Space Sci. 259, 415 (1998).

22. Chen, H. W. et al. Nature 398, 586 (1999).

23. Chen, H. W. et al. Nature 408, 562 (2000).

24. Stern, D. et al. Nature 408. 5602000.

25. Basu, D. Astrophys. Letts. Commun. 40, 157 (2001).

26. Wampler, E. and Oke, J. Astrophys. J. 148, 695 (1967).

27. McDowell, J. et al. Astrophys. J. 450, 585 (1995).

28. Jannuzi, B. Astrophys. J. Suppl. 118, 1 (1998).

29. Basu, D. Phys. Scr. 69, 427 (2004).

30. Basu, D. and Haque-Copilah, S. Phys. Scr. 63, 425 (2001).

31. Basu, D. Astron. J. 131, 1231 (2006).

32. Basu, D. J. Astrophys. Astron. 27, 381 (2006).

33. Kulkarni, S. et al. Nature 393, 35 (1998).

34. Basu, D. Astrophys. Letts. Commun. 40, 225 (2001).

35. Riess, A. et al. Astron. J. 116, 1009 (1998).

36. Basu, D. Mod. Phys. Letts. A 15, 2357 (2000).

37. Basu, D. Astron. Nachr. 327, 724 (2006).

38. Basu, D. Astrophys. Letts. 22, 139 (1982).

39. Moller, P. et al. Astron. Astrophys. 296, L21 (2002).

40. Mirabell, N. et al. Astrophys. J. 595, 935 (2003).

41. Mirabell, N. et al. Astrophys. J. 587, 128 (2003).

42. Berger, E. et al. Astrophys. J. 587, L5 (2003). 
43. Masetti, N. et al. Astron. Astrophys. 465, 481 (2003).

44. Masetti, N.et al. Astron. Astrophys. 374, 382 (2001).

45. Metzger, M. Nature 387, 878 (1997).

46. Basu, D. Nat. Phys. Sci. 241, 159 (1973).

47. Basu, D. The Observatory 93, 229 (1973).

48. Smith, H. et al. Astrophys. J. 228, 369 (1979).

49. Mayor, D. and York, D. Astrophys. J. 319, L45 (1987).

50. Ptak, A. et al. Astron. J. 113, 1286 (1997).

51. Basu, D. et al. Astron. Astrophys. 272, 417 (1993).

52. Capetti, A. et al. Astron. Astrophys. 431, 465 (2005).

53. Saslaw, W. et al. Astrophys. J. 190, 253 (1974).

54. Valtonen, M. Astron. Astrophys. 46, 429 (1976).

55. Valtonen, M. Astron. Astrophys. 46, 435 (1976).

56. Valtaoja, L. et al. Astrophys. J. 343, 47 (1989).

57. Komossa, S. at al. Astrophys. J. 582, L15 (2003).

58. Valtonen, M. et al. Astrophys. J. 643, L9 (2006).

59. Boronson, T. and Lauer, T. Nature 458, 53 (2009).

60. Mikkola, S. and Valtonen, M. Astrophys. J. 348, 412 (1990).

61. Haehnelt, M. et al. Mon. Not. Roy. Astron. Soc. 366, L22 (2006).

62. Rees, M. and Saslaw, W. Mon. Not. Roy. astron. Soc. 171, 53 (1975).

63. Lin, D. and Saslaw, W. Astrophys. J. 217, 958 (1977).

64. De Young, D. Astrophys. J. 211, 329 (1977). 
65. Rees, M. Ann. Rev. Astron. Astrophys. 22, 471 (1984).

66. Osterbrock, D. and Mathews, W. Ann. Rev. Astron. Astrophys. 24, 171 (1986).

67. Valtonen, M. and Basu, D. J. Astrophys. Astron. 12, 91 (1991).

68. Carr, B. Comm. Astrophys. 7, 161 (1978).

69. Dressler, A., et al. Astrophys. J. 404, L45 (1993).

70. Francis, P., et al. Astrophys. J. 457, 490 (1996).

71. Giavalisco M., et al. Astrophys. J. 425, L5 (1994). 